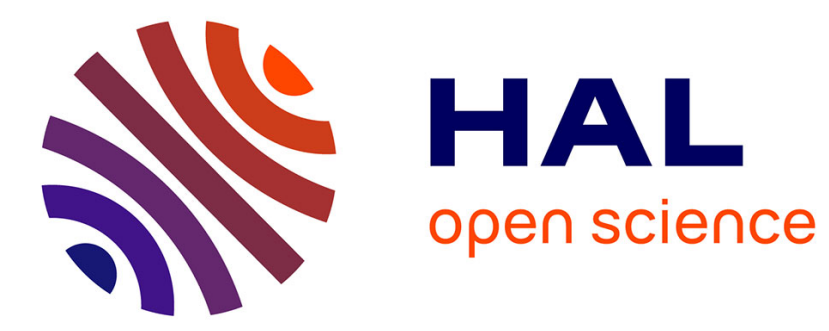

\title{
Application de la méthode de rétrodiffusion des électrons à la mesure en continu des faibles épaisseurs de revêtements légers sur métaux ou alliages légers
}

\author{
G.R. Roche, J.F. Deveau, P.J. Cunat, P. Levy, J. Boin, C. Riss
}

\section{- To cite this version:}

G.R. Roche, J.F. Deveau, P.J. Cunat, P. Levy, J. Boin, et al.. Application de la méthode de rétrodiffusion des électrons à la mesure en continu des faibles épaisseurs de revêtements légers sur métaux ou alliages légers. Revue de Physique Appliquée, 1970, 5 (2), pp.317-320. 10.1051/rphysap:0197000502031700 . jpa-00243394

\section{HAL Id: jpa-00243394 https://hal.science/jpa-00243394}

Submitted on 1 Jan 1970

HAL is a multi-disciplinary open access archive for the deposit and dissemination of scientific research documents, whether they are published or not. The documents may come from teaching and research institutions in France or abroad, or from public or private research centers.
L'archive ouverte pluridisciplinaire HAL, est destinée au dépôt et à la diffusion de documents scientifiques de niveau recherche, publiés ou non, émanant des établissements d'enseignement et de recherche français ou étrangers, des laboratoires publics ou privés. 


\title{
APPLICATION DE LA MÉTHODE DE RÉTRODIFFUSION DES ÉLECTRONS A LA MESURE EN CONTINU DES FAIBLES ÉPAISSEURS DE REVÊTEMENTS LÉGERS SUR MÉTAUX OU ALLIAGES LÉGERS
}

\author{
par G. R. ROCHE, J. F. DEVEAU, P. J. CUNAT, \\ Laboratoire de Physique Nucléaire, Faculté des Sciences de Clermont-Ferrand \\ P. LEVY, J. BOIN, \\ Institut Universitaire de Technologie, Montluçon \\ C. RISS, \\ Laboratoires de Recherches, C. E. G. E. D. U. R., Issoire
}

(Reçu le 25 novembre 1969)

\begin{abstract}
Résumé. - Le problème envisagé concerne la mesure en continu des faibles épaisseurs de revêtements organiques sur métaux ou alliages légers. Nous étudions les caractéristiques d'une jauge à rétrodiffusion $\beta$ opérant sous atmosphère d'hélium. Nous montrons que le fait de travailler sous hélium améliore les résultats de façon très importante. L'influence de l'énergie de la source radio-active et du numéro atomique du revêtement sont examinés. Les résultats obtenus concernent également la précision, le temps de mesure ainsi que la surface de l'échantillon sous investigation.

Abstract. - This paper deals with the continuous thickness measurement of thin organic coatings on light metals or alloys. The characteristics of a beta-ray backscattering gauge operating in helium are studied therein. We show that results are very much improved by working in helium. The influences of the beta radiation energy and of the atomic number of the coating are examined. Results are also given about the accuracy and the times of measurement, as well as the surface of the sample tested.
\end{abstract}

I. Introduction. - La méthode de rétrodiffusion des électrons est fréquemment utilisée pour la mesure des épaisseurs. Le plus souvent, les publications ou documentations techniques [1, 2, 3] font allusion aux revêtements de numéro atomique élevé (par exemple, dépôt électrolytique) sur métaux ou alliages lourds, la méthode étant d'autant plus précise que les numéros atomiques du revêtement et du support sont différents.

Le problème que nous avons abordé concerne la mesure en continu des faibles épaisseurs de revêtements tels que vernis ( $Z$ faible, épaisseurs $\lesssim 20 \mu \mathrm{m}$ ) sur aluminium ou alliage léger. Une étude bibliographique nous a montré que le domaine sous investigation était à la limite des performances des appareils existants. En effet, dans notre cas, nous devons utiliser des énergies assez faibles, de l'ordre de $100 \mathrm{keV}$ (Cf. § II) ; les appareils actuellement commercialisés opèrent dans l'air et des énergies aussi faibles sont très rapidement absorbées. On peut donc penser travailler dans le vide ; la méthode est évidemment applicable pour un appareil de laboratoire.

Mais notre problème doit déboucher sur le contrôle en continu sur une chaîne de vernissage, ce qui n'est pas réalisable pratiquement en opérant sous vide. Nous avons donc été conduits à prendre une solution intermédiaire qui consiste à opérer sous atmosphère d'hélium, choix original, à notre connaissance.

La présente communication donne les résultats que nous avons pu obtenir en laboratoire.

II. Energie du rayonnement. - Lorsqu'on opère sur un ensemble constitué par un matériau de base et un revêtement dont on désire mesurer l'épaisseur, il y a lieu tout d'abord d'éliminer l'influence du matériau de base. Pour cela, il faut que l'épaisseur de celui-ci soit supérieure ou égale à l'épaisseur correspondant à la saturation. Dans ces conditions, les courbes donnant la variation de l'intensité rétrodiffusée en fonction de l'épaisseur du revêtement, sont représentées sur la figure 1.

Pour fixer les ordres de grandeur, nous considérons le cas d'une couche de vernis de $10 \mu \mathrm{m}$ ( $Z$ moyen égal à 6 , masse volumique voisine de $1 \mathrm{~g} \mathrm{~cm}^{-3}$ ) déposée sur de l'aluminium $(Z=13)$. La courbe correspondante est alors celle de la figure $1 b$ et on a :

$$
I_{R} \# \frac{I_{B}}{2}
$$

$e_{\mathrm{s}} \sim 20 \mu \mathrm{m}$ pour une énergie $\beta$ de $100 \mathrm{keV}$,

$e_{\mathrm{s}} \sim 35 \mu \mathrm{m}$ pour une énergie $\beta$ de $200 \mathrm{keV}$. 

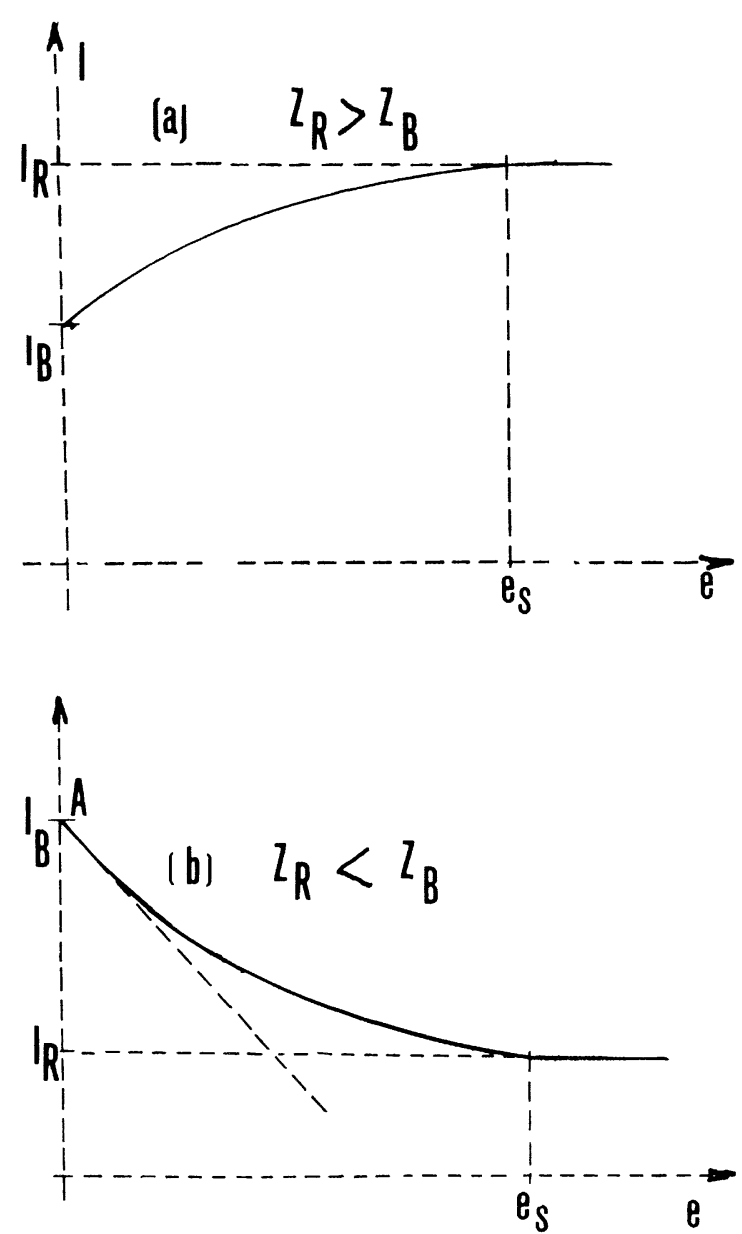

FIG. 1. - Variation de l'intensité rétrodiffusée $I$ en fonction de l'épaisseur $e$ du revêtement : $Z_{\mathrm{R}}=$ numéro atomique du revêtement; $Z_{\mathrm{B}}=$ numéro atomique du matériau de base ; $I_{\mathrm{R}}=$ intensité rétrodiffusée à saturation par le revêtement; $I_{\mathrm{B}}=$ intensité rétrodiffusée à saturation par le matériau de base; $e_{\mathrm{S}}=$ épaisseur du revêtement correspondant à la saturation.

Si on assimile la courbe précédente à sa tangente au point $\mathrm{A}$, on trouve que $10 \mu \mathrm{m}$ de vernis fournissent une variation d'intensité de $60 \%$ pour une énergie $\beta$ de $100 \mathrm{keV}$ et de $30 \%$ pour une énergie $\beta$ de $200 \mathrm{keV}$.

Nous sommes donc conduits à utiliser les radioéléments suivants :

${ }^{14} \mathrm{C}$ : énergie $=0,15 \mathrm{MeV}$; période $=5600 \mathrm{~A}$,

${ }^{99} \mathrm{Tc}:$ énergie $=0,29 \mathrm{MeV}$; période $=2,1 \times 10^{5} \mathrm{~A}$.

Remarquons que la condition relative à l'épaisseur du matériau de base est très facilement réalisée car $250 \mu \mathrm{m}$ d'aluminium correspondent à la saturation pour toute énergie $\beta$ inférieure à $300 \mathrm{keV}$.

III. Résultats expérimentaux. - Le dispositif de mesure est extrêmement simple. Il comprend essentiellement (Fig. 2) :

- une cloche à Hélium ouverte dans sa partie inférieure ;

- la ou les sources radio-actives ;
- le détecteur (compteur Geiger-Müller à fenêtre mince ou scintillateur plastique associé à un photomultiplicateur ; les mesures avec scintillateur et photomultiplicateur sont généralement meilleures) ;

- l'échantillon (feuille d'aluminium de $250 \mu \mathrm{m}$ d'épaisseur recouverte de vernis).

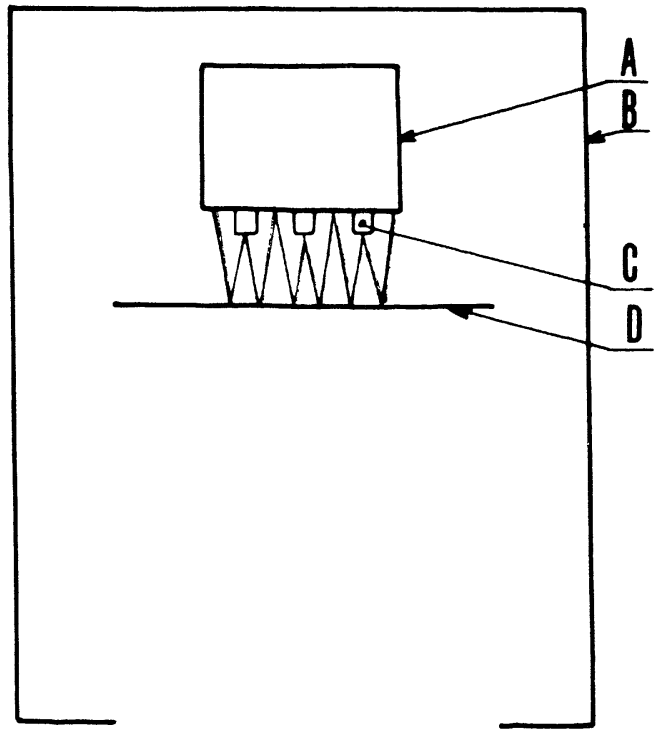

FIG. 2. - Schéma de principe du dispositif expérimental : $\mathrm{A}$ : détecteur; $\mathrm{B}$ : cloche à hélium; $\mathrm{C}$ : sources radioactives; $\mathrm{D}$ : échantillon.

Le résultat le plus important est donné par la figure 3, où nous avons porté le taux de comptage du détecteur en fonction de l'épaisseur de vernis $(Z=6)$ dans les deux cas où on opère dans l'air et dans l'hélium.

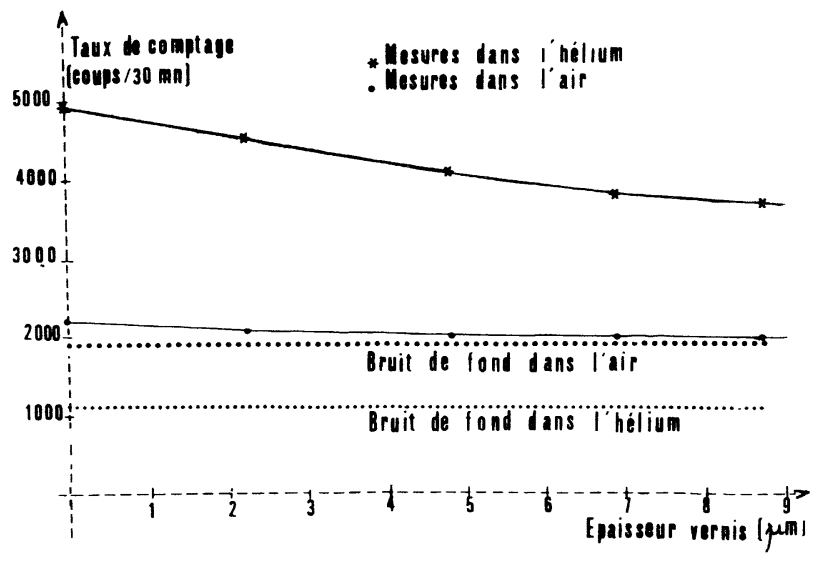

FIG. 3. - Influence du milieu ambiant. Source : ${ }^{14} \mathrm{C}$. Détecteur : compteur G. M.

Le comptage en l'absence d'échantillon («bruit de fond ») a également été relevé.

On constate sur cette figure une amélioration radicale en opérant dans l'hélium. Le rapport du comptage avec échantillon sans vernis au comptage sans échantillon est de 4,5 dans l'hélium contre 1,1 dans l'air. 
Les courbes de la figure 4 donnent le taux de comptage en fonction de l'épaisseur de vernis $(Z=6)$ dans le cas où on utilise des sources de ${ }^{14} \mathrm{C}$ ou de ${ }^{99}$ Tc. Le résultat est évidemment meilleur avec la source de ${ }^{14} \mathrm{C}$.

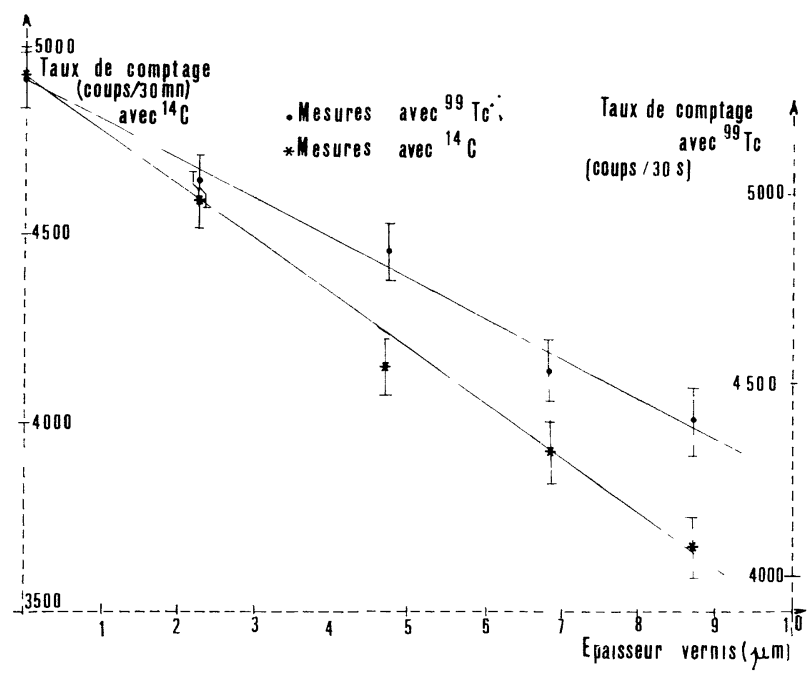

FIG. 4. - Influence de l'énergie de la source radioactive. Détecteur : compteur G. M. Milieu ambiant: Hélium.

Enfin, les courbes des figures 5 et 6 montrent les résultats obtenus avec un vernis de numéro atomique égal à 6 et avec un vernis pigmenté ( $Z$ moyen voisin de 10). Les courbes ont été déterminées avec une précision bien meilleure que dans les cas précédents. Leur allure est un peu différente en particulier, la zone linéaire ne s'étend pas au domaine des épaisseurs inférieures à $2 \mu \mathrm{m}$.

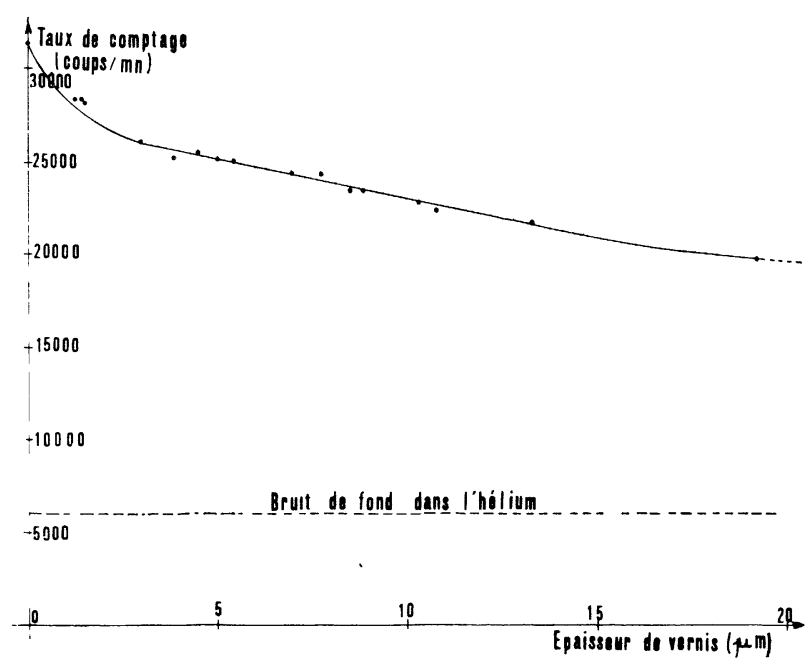

FIG. 5. - Variation du taux de comptage en fonction de l'épaisseur de vernis $(Z=6)$. Détecteur : scintillateur et photomultiplicateur. Radioélément : ${ }^{14} \mathrm{C}$. Milieu ambiant : Hélium.

IV. Caractéristique des sondes de mesure à rétrodiffusion. - Sur la figure 4 nous relevons une variation relative de comptage de $22 \%$ pour une variation de

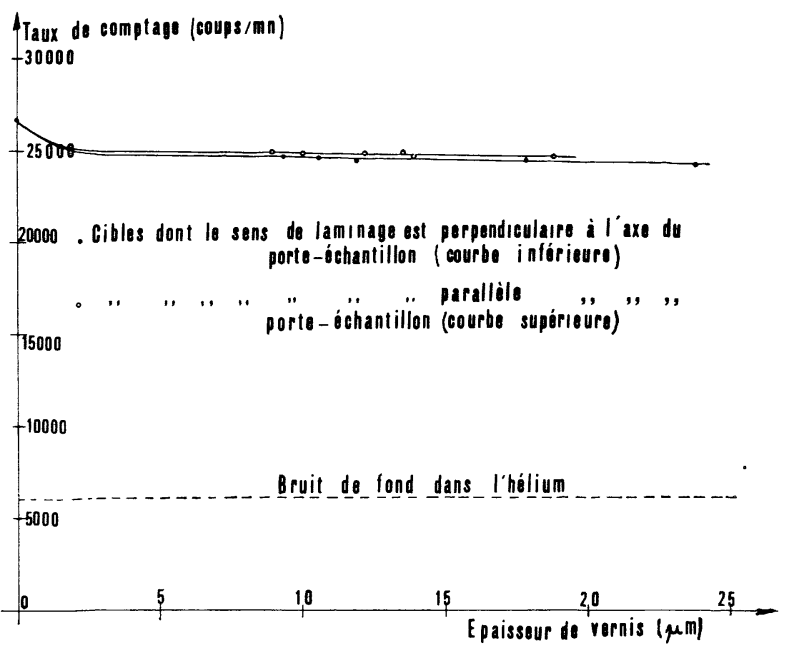

Fig. 6. - Variation du taux de comptage en fonction de l'épaisseur de vernis pigmenté $(Z=10)$. Détecteur : scintillateur et photomultiplicateur. Radioélément : ${ }^{14} \mathrm{C}$. Milieu ambiant : Hélium.

$10 \mu \mathrm{m}$ de l'épaisseur de vernis dans le cas du ${ }^{99} \mathrm{Tc}$ et de $35 \%$ dans le cas $d u{ }^{14} \mathrm{C}$. Ces valeurs sont en accord avec les ordres de grandeur obtenus dans le paragraphe II.

De façon générale, nous définissons quantitativement les performances d'une jauge d'épaisseur à rétrodiffusion en introduisant le coefficient caractéristique :

$$
\zeta=\frac{1}{I \rho} \cdot \frac{\Delta I}{\Delta e}=\frac{1}{I} \cdot \frac{\Delta I}{\Delta m}
$$

où $\Delta I$ représente la variation du taux de comptage $I$ consécutif à une augmentation d'épaisseur $\Delta e$, soit une augmentation $\Delta m$ de masse superficielle, $\rho$ étant la masse volumique.

Dans le cas de nos mesures avec le ${ }^{14} \mathrm{C}$, nous obtenons des valeurs comprises entre $\zeta=0,19 \mathrm{mg}^{-1} . \mathrm{cm}^{2}$ (partie linéaire de la courbe de la Fig. 4) et

$$
\zeta=0,35 \mathrm{mg}^{-1} \cdot \mathrm{cm}^{2} \text { (Fig. 3) . }
$$

Plusieurs jauges d'épaisseur par rétrodiffusion sont actuellement commercialisées. Nous avons retenu deux types : l'un, le Béta 750 est fourni par la Société Johnson-Matthey et Co [2], l'autre par la Société Philips [1]. Compte tenu des données que nous avons pu obtenir sur ces appareils, il semble que le Béta 750 soit caractérisé par le coefficient $\zeta=0,036 \mathrm{mg}^{-1} . \mathrm{cm}^{2}$ et la jauge Philips par $\zeta=0,11 \mathrm{mg}^{-1} \cdot \mathrm{cm}^{2}$.

Ces valeurs du paramètre $\zeta$ sont inférieures à celles que nous avons obtenues. De plus, nous opérons sur des matériaux de numéro atomique plus faible, ce qui est défavorable. Dans notre cas, revêtement: $Z=6$ (vernis), base : $Z=13$ (aluminium). Dans le cas de la jauge Philips, revêtement: $Z=50$ (étain), base : $Z=26$ (fer). Dans le cas du Béta 750, revêtement $Z=79$ (or), base : $Z=29$ (cuivre). 
V. Temps de mesure. - Un autre paramètre est fondamental pour l'utilisateur: le temps de mesure. Ce paramètre est fonction de l'activité de la source de l'angle solide source-échantillon-détecteur, et de l'efficacité du détecteur, pour une précision de mesure d'épaisseur imposée.

Ces trois facteurs étant optimisés, le temps de mesure est encore fonction du coefficient $\zeta$.

Soit $\Delta m$ la précision souhaitée sur la mesure d'épaisseur ; supposons que l'erreur soit uniquement statistique; prenons :

$$
\Delta N=x \sqrt{N}
$$

avec $N=$ comptage ; $x=1,2$ ou 3 suivant l'intervalle de confiance désiré. On a :

$$
\frac{\Delta N}{N}=\frac{\Delta I}{I} .
$$

On obtient à l'aide de (1) et (2) :

$$
N=\frac{x^{2}}{\zeta^{2} \cdot \Delta m^{2}}
$$

Or : $N=k \cdot A . t, A$ étant l'activité de la source, $t$ le temps et $k$ un coefficient de proportionnalité dépendant de la géométrie et de l'efficacité de détection.

Il en résulte que, pour une précision imposée, le temps de mesure est inversement proportionnel à $\zeta^{2}$, toutes choses égales par ailleurs.

Dans l'expérience correspondant à la figure 4, le taux de comptage moyen est de 23000 coups par minute. La formule (3) avec $x=2$ donne $N=180000$ pour une précision $\Delta e= \pm 0,25 \mu \mathrm{m}$. Le temps de mesure pour obtenir cette précision est de 8 minutes.

Dans cette expérience, on disposait de 5 sources de
$100 \mu \mathrm{Ci}$ de ${ }^{14} \mathrm{C}$ placées entre l'échantillon et un scintillateur de $80 \mathrm{~cm}^{2}$ de surface [4]. On peut utiliser jusqu'à 25 sources, ce qui réduit le temps de mesure à 1 ou 2 minutes pour obtenir la précision de $\pm 0,25 \mu \mathrm{m}$.

VI. Conclusion. - Le matériau de base étant l'aluminium $(Z=13)$, il est possible de mesurer avec une précision de $\pm 0,25 \mu \mathrm{m}$ des épaisseurs de revêtement inférieures à $20 \mu \mathrm{m}$ dans le cas où le numéro atomique du revêtement est voisin de 6 . Le temps de comptage est de 1 ou 2 minutes. La surface d'échantillon explorée est de l'ordre de $100 \mathrm{~cm}^{2}$.

La méthode devient inapplicable pour des revêtements de numéro atomique voisin de 10. Dans les mêmes conditions que précédemment, il faudrait un temps de mesure 100 fois plus long pour obtenir la même précision.

On peut espérer que la méthode est valable d'une façon générale lorsque la différence entre les numéros atomiques du revêtement et du support (aluminium) est supérieur à 4 ou 5 , en plus ou en moins.

Ces résultats nous ont permis d'envisager une application industrielle dans le contrôle en continu sur une chaîne de vernissage, la mesure pouvant alors être considérée comme ponctuelle [5].

Quelques problèmes technologiques restent à résoudre, le principal étant le stockage de l'hélium sous une cloche ouverte à sa partie inférieure. Une étude est en cours et sera publiée ultérieurement [4].

Nous remercions vivement MM. L. Avan, Profesreur à la Faculté des Sciences de Clermont-Ferrand et M. Renouard, Directeur du Laboratoire de Recherches C. E. G. E. D. U. R. à Issoire qui nous ont proposé l'étude décrite dans cette publication.

\section{Bibliographie}

[1] ( Jauge de mesure d'épaisseur » (1967), Documentation Philips; LAFAY, Electronique Industrielle, 1967, 819-821.

[2] Béta 750, Documentation Johnson-Matthey \& Co.

[3] Bétascope, Documentation Fischer.
[4] ((Etude technologique de l'application industrielle ): à paraître.

[5] Brevet B 1479 déposé le 9.10.1969 sous le No PV 69 34571 par la Société dite : C. E. G. E. D. U. R. G. P. 